Rustam Ibadov • Burkhard Kleihaus • Jutta Kunz •

Sardor MurodoviD

\title{
Wormhole solutions with NUT charge in higher curvature theories
}

Received: 18 October 2021 / Accepted: 28 October 2021 / Published online: 22 November 2021

(C) The Author(s) 2021

\begin{abstract}
We present wormholes with a Newman-Unti-Tamburino (NUT) charge that arise in certain higher curvature theories, where a scalar field is coupled to a higher curvature invariant. For the invariants we employ (i) a Gauss-Bonnet term and (ii) a Chern-Simons term, which then act as source terms for the scalar field. We map out the domain of existence of wormhole solutions by varying the coupling parameter and the scalar charge for a set of fixed values of the NUT charge. The domain of existence for a given NUT charge is then delimited by the set of scalarized nutty black holes, a set of wormhole solutions with a degenerate throat and a set of singular solutions.
\end{abstract}

\section{Mathematics Subject Classification (2000) 83D05}

\section{Introduction}

Wormholes are intriguing solutions of numerous theories of gravity. In General Relativity the presence of some form of exotic matter is required to construct traversable Lorentzian wormholes, since the energy conditions must be violated (see, e.g., $[32,34,37]$ ). The simplest possibility here is to employ a phantom scalar field, i.e., a field whose kinetic term has the opposite sign as compared to an ordinary scalar field. Such phantom fields have been already employed decades ago by Ellis and Bronnikov, when constructing wormhole solution in General Relativity $[6,14,15,31]$.

In alternative theories of gravity, however, classical Lorentzian wormholes may arise without the need for exotic matter. The reason is, that the gravitational interaction itself can give rise to additional terms, that may be interpreted as contributing to an effective stress energy tensor on the right hand side of the Einstein field equations, which then may lead to violation of the energy conditions. Thus it is the modified gravitational interaction itself which provides the necessary violation of the energy conditions (see, e.g., $[7,17-20,22,27,28,33])$.

R. Ibadov $\cdot$ S. Murodov ( $\square)$

Department of Theoretical Physics and Computer Science, Samarkand State University, Samarkand 140104, Uzbekistan

E-mail: mursardor@mail.ru

R. Ibadov

E-mail: ibrustam@mail.ru

B. Kleihaus - J. Kunz

Institute of Physics, University of Oldenburg, 26111 Oldenburg, Germany

E-mail: b.kleihaus@uni-oldenburg.de

J. Kunz

E-mail: jutta.kunz@uni-oldenburg.de 
In recent years, alternative gravity theories have received much attention $[3,16,35,38]$. To a large extent this interest has been driven by the quest to resolve various cosmological issues. However, alternative theories of gravity may also lead to interesting predictions for compact objects in the strong gravity regime, where the advent of gravitational wave multi-messenger astronomy has opened a new window into the universe with the new observational possibilities and challenges.

Among the numerous alternative gravitational theories we consider higher curvature theories very attractive, where a scalar field is coupled to a higher curvature invariant. On the one hand higher curvature terms arise under various circumstances, when quantum gravity theories are considered, and on the other hand their coupling constants may still be large, thus leading to interesting potentially observable effects in the strong gravity regime, since constraints from the solar system or binary pulsars may easily be satisfied.

Here, we consider two such theories: (i) Einstein-scalar-Gauss-Bonnet (EsGB) theory and (ii) Einsteinscalar-Chern-Simons (EsCS) theory. In both cases we choose the same type of coupling function of the scalar field to the invariant, namely, a function quadratic in the scalar field. When such a quadratic coupling is chosen, a vacuum solution of General Relativity supplemented with vanishing scalar field is also a solution of EsGB and EsCS theory. Thus the Schwarzschild black hole and the Kerr black hole remain solutions of these alternative gravity theories. However, these theories may possess additional black hole solutions with a finite scalar field, i.e., spontaneously scalarized black holes.

In the case of EsGB theory spontaneously, scalarized black holes were first obtained in the static spherically symmetric case $[1,13,36]$ and later generalized to include rotation $[4,8,9,12,21]$. Here the Gauss-Bonnet (GB) invariant acts as an effective mass term in the scalar field equation, that may induce a tachyonic instability of the Schwarzschild or Kerr black holes.

In the case of EsCS theory, there are no spontaneously scalarized static spherically symmetric black holes. The reason is that the Chern-Simons (CS) invariant vanishes for the Schwarzschild solution. So no tachyonic instability will be induced in this case. For the CS invariant to contribute one would need the presence of a parity-odd source. Such a source would be rotation [11,39]; however, the construction of rotating spontaneously scalarized EsCS black holes will be technically rather challenging.

Therefore, as a first step towards obtaining rotating spontaneously scalarized black holes in EsCS theory, a by far simpler case has been studied in [5]. Here a NUT charge has been introduced to obtain a parity odd source term, but retain a system of ordinary differential equations, since the angular dependence factorizes. The CS invariant can then act as an effective mass term in the scalar field equation and induce a tachyonic instability of the Schwarzschild black holes. A NUT charge is, in fact, quite intriguing. It gives, for instance, rise to a Misner string on the polar axis and implies the presence of closed time-like curves (see, e.g., [26] and references therein).

Here, we consider wormholes with NUT charge in (i) EsGB theory and (ii) EsCS theory, recalling and extending previous results $[2,23,24]$. Since one boundary of the domain of existence of wormholes in such theories is typically given by the corresponding set of black holes, we follow closely Brihaye et al. [5], who have obtained the sets of spontaneously scalarized EsGB and EsCS black holes with NUT charge. The presence of the NUT charge does not lead to asymptotically flat solutions in the usual sense. However, the asymptotic fall-off of the functions is of the usual type of an asymptotically flat spacetime.

This paper is organized as follows: we first present the action for both theories and their equations of motion. Then, we discuss the conditions for the presence of a throat. We next determine the boundary conditions, the junction conditions and the energy conditions. Subsequently, we present our results for (i) EsGB wormholes and (ii) EsCS wormholes with NUT charge. We then end with our conclusions.

\section{Action and equations of motion}

We employ the effective action for Einstein-scalar-higher curvature invariant theories as presented in Brihaye et al. [5] (with geometrized units with $G=c=1$ ):

$$
S=\frac{1}{16 \pi} \int\left[R-\frac{1}{2} \partial_{\mu} \phi \partial^{\mu} \phi+F(\phi) \mathcal{I}(g)\right] \sqrt{-g} \mathrm{~d}^{4} x,
$$

where $R$ is the curvature scalar, $\phi$ denotes a massless scalar field without self-interaction, coupled with coupling function $F(\phi)$ to an invariant $\mathcal{I}(g)$. For the coupling function $F(\phi)$ we choose a quadratic $\phi$-dependence with coupling constant $\alpha$ :

$$
F(\phi)=\alpha \phi^{2} .
$$


This is the simplest choice that leads to spontaneous scalarization of black holes. We emphasize, that we follow the conventions of Brihaye et al. [5], since we would like to compare with their results. Obviously, any prefactor of the kinetic term in the action Eq. (1) can be absorbed by re-definitions of the scalar field $\phi$ and parameter $\alpha$ of the coupling function $F(\phi)=\alpha \phi^{2}$.

For the invariant $\mathcal{I}(g)$ we make the following choices: (i) we choose the Gauss-Bonnet term:

$$
\mathcal{I}(g)=R_{\mathrm{GB}}^{2}=R_{\mu \nu \rho \sigma} R^{\mu \nu \rho \sigma}-4 R_{\mu \nu} R^{\mu \nu}+R^{2},
$$

and (ii) we choose the Chern-Simons term:

$$
\mathcal{I}(g)=R_{\mathrm{CS}}^{2}={ }^{*} R_{\nu}^{\mu}{ }^{\rho \sigma} R^{\nu}{ }_{\mu \rho \sigma} .
$$

Here, we have employed the Hodge dual of the Riemann-tensor ${ }^{*} R^{\mu}{ }_{\nu}{ }^{\rho \sigma}=\frac{1}{2} \eta^{\rho \sigma \kappa \lambda} R^{\mu}{ }_{\nu \kappa \lambda}$ which is defined with the 4-dimensional Levi-Civita tensor $\eta^{\rho \sigma \kappa \lambda}=\epsilon^{\rho \gamma \sigma \tau} / \sqrt{-g}$. We note that both invariants are topological in four dimensions. However, their coupling to the scalar field $\phi$ lets them contribute to the equations of motion.

The coupled sets of field equations are then obtained by variation of the action (1) with respect to the scalar field and the metric:

$$
\begin{aligned}
& \nabla^{\mu} \nabla_{\mu} \phi+\frac{\mathrm{d} F(\phi)}{\mathrm{d} \phi} \mathcal{I}=0, \\
& G_{\mu \nu}=\frac{1}{2} T_{\mu \nu}^{(\text {eff })} .
\end{aligned}
$$

Here, $G_{\mu \nu}$ is the Einstein tensor, as usual, while $T_{\mu \nu}^{(\text {eff })}$ denotes the resulting effective stress energy tensor:

$$
T_{\mu \nu}^{(\mathrm{eff})}=T_{\mu \nu}^{(\phi)}+T_{\mu \nu}^{(\mathcal{I})},
$$

consisting of the standard scalar field contribution:

$$
T_{\mu \nu}^{(\phi)}=\left(\nabla_{\mu} \phi\right)\left(\nabla_{\nu} \phi\right)-\frac{1}{2} g_{\mu \nu}\left(\nabla_{\rho} \phi\right)\left(\nabla^{\rho} \phi\right),
$$

and a gravitational contribution from the respective higher curvature invariant $\mathcal{I}(g)$. For the two above invariants we obtain (i)

$$
T_{\mu \nu}^{(G B)}=\left(g_{\rho \mu} g_{\lambda \nu}+g_{\lambda \mu} g_{\rho \nu}\right) \eta^{\kappa \lambda \alpha \beta *} R_{\alpha \beta}^{\rho \gamma} \nabla_{\gamma} \nabla_{\kappa} F(\phi),
$$

and (ii)

$$
T^{(C S) \mu \nu}=-8\left[\nabla_{\rho} F(\phi)\right] \epsilon^{\rho \sigma \tau(\mu} \nabla_{\tau} R^{\nu)}{ }_{\sigma}+\left[\nabla_{\rho} \nabla_{\sigma} F(\phi)\right]^{*} R^{\sigma(\mu \nu) \rho} .
$$

To obtain wormhole solutions with a NUT charge $N$, we assume the line element to be of the form:

$$
\mathrm{d} s^{2}=-\mathrm{e}^{f_{0}}(\mathrm{~d} t-2 N \cos \theta \mathrm{d} \varphi)^{2}+\mathrm{e}^{f_{1}}\left[\mathrm{~d} r^{2}+r^{2}\left(\mathrm{~d} \theta^{2}+\sin ^{2} \theta \mathrm{d} \varphi^{2}\right)\right] .
$$

With this ansatz the angular dependence factorizes, and all three functions, the two metric functions $f_{0}$ and $f_{1}$ and the scalar field function $\phi$, depend only on the radial coordinate $r$.

Insertion of the above ansatz (11) for the metric and the scalar field into the scalar field equation (5) and the Einstein equations (6) with effective stress-energy tensor (7) leads to five coupled, nonlinear ordinary differential equations (ODEs). However, these are not all independent, and one ODE can be treated as a constraint. We thus retain three coupled ODEs of second order to be solved numerically. We remark that in case (i) we can even obtain one first order and two second order ODEs.

The field equations possess an invariance under the scaling transformation:

$$
r \rightarrow \chi r, \quad N \rightarrow \chi N, \quad t \rightarrow \chi t, \quad F \rightarrow \chi^{2} F
$$

with constant $\chi>0$. 


\section{Throat and boundary conditions}

To obtain wormhole solutions, the presence of a throat is mandatory. We, therefore, introduce the circumferential (or spherical) radius:

$$
R_{C}=\mathrm{e}^{\frac{f_{1}}{2}} r
$$

of the spacetime. When the circumferential radius has a single finite extremum, a minimum, this corresponds to the single throat of the wormhole, since the throat is a surface of minimal area. While wormholes with more extrema also exist, which then feature an equator surrounded by a double throat, we here focus on single throat wormholes.

We now require the presence of an extremum of the circumferential radius at some $r=r_{0}$, to obtain the first set of boundary conditions. This leads to

$$
\left.\frac{\mathrm{d} R_{C}}{\mathrm{~d} r}\right|_{r=r_{0}}=\left.0 \Longleftrightarrow \frac{\mathrm{d} f_{1}}{\mathrm{~d} r}\right|_{r=r_{0}}=-\frac{2}{r_{0}} .
$$

Consequently, we will refer to the two-dimensional submanifolds defined by $r=r_{0}$ and $t=$ const. as the throat of the wormholes.

The presence of the NUT charge endows these wormholes with an interesting feature: the throat metric of the wormholes with NUT charge:

$$
\mathrm{d} s_{\mathrm{th}}^{2}=\mathrm{e}^{f_{1}\left(r_{0}\right)} r_{0}^{2}\left(\mathrm{~d} \theta^{2}+\left[\sin ^{2} \theta-\frac{4 N^{2}}{r_{0}^{2}} \mathrm{e}^{f_{0}\left(r_{0}\right)-f_{1}\left(r_{0}\right)} \cos ^{2} \theta\right] \mathrm{d} \varphi^{2}\right)
$$

changes its signature, when the coefficient of $\mathrm{d} \varphi^{2}$ changes sign. This happens at the critical angles $\theta_{c}$ and $\pi-\theta_{c}$, where $\theta_{c}$ is obtained by requiring $\operatorname{det}\left(g_{\text {th }}\right) \geq 0$, and thus $\theta_{c} \leq \theta \leq \pi-\theta_{c}$ with

$$
\theta_{c}=\left.\arctan \left(\frac{2|N|}{r_{0}} \mathrm{e}^{\frac{f_{0}-f_{1}}{2}}\right)\right|_{r_{0}} .
$$

The metric has positive signature, as required for a two-dimensional Riemannian surface, only for $\theta_{c} \leq \theta \leq$ $\pi-\theta_{c}$. The signature change is, however, not a surprise for a spacetime with a NUT charge, since a NUT charge leads to a non-causal structure of a spacetime and allows for closed time-like curves.

The second set of boundary conditions is obtained by imposing the usual fall-off as for asymptotically flat spacetimes and thus the usual boundary conditions for $r \rightarrow \infty$ [5]. Thus the metric functions and the scalar field satisfy the asymptotic expansions:

$$
\begin{aligned}
f_{0} & =-\frac{2 M}{r}+\mathcal{O}\left(r^{-3}\right), \\
f_{1} & =\frac{2 M}{r}+\mathcal{O}\left(r^{-2}\right), \\
\phi & =\phi_{\infty}-\frac{D}{r}+\mathcal{O}\left(r^{-3}\right),
\end{aligned}
$$

where the constants $M$ and $D$ denote the mass and the scalar charge of the wormholes, respectively, while $\phi_{\infty}$ denotes the asymptotic value of the scalar field. All higher order terms in the expansion can be expressed in terms of the constants $M, D$ and $\phi_{\infty}$. A solution is, therefore, uniquely determined by these three constants (and, of course, the parameters of the theory). Since we follow Brihaye et al. [5] in order to compare with their spontaneously scalarized black hole solutions, we need to also employ their chosen asymptotic value $\phi_{\infty}=0$.

\section{Junction conditions}

Our aim is to obtain wormholes, which are symmetric with respect to the throat. Inspection of the functions at the throat shows, that if the solutions would be simply continued to the other side of the throat, the solutions would not be symmetric. Worse, however, is that further integration beyond the throat will lead to a curvature singularity, and asymptotic infinity on that side cannot be reached. Therefore, we cut the solutions at the throat 
and paste a symmetric copy on the other side of the throat. To have continuous and differentiable wormholes, we, therefore, need to impose junction conditions at the throat.

To that end we introduce the radial coordinate $\eta$

$$
\eta=r_{0}\left(\frac{r}{r_{0}}-\frac{r_{0}}{r}\right)
$$

where $r_{0}$ is a constant, and define the constant $\eta_{0}$ via $\eta_{0}=2 r_{0}$. We then reexpress the metric in terms of the new radial coordinate $\eta$ :

$$
\mathrm{d} s^{2}=-\mathrm{e}^{f_{0}}(\mathrm{~d} t-2 N \cos \theta \mathrm{d} \varphi)^{2}+\mathrm{e}^{F_{1}}\left[\mathrm{~d} \eta^{2}+\left(\eta^{2}+\eta_{0}^{2}\right)\left(\mathrm{d} \theta^{2}+\sin ^{2} \theta \mathrm{d} \varphi^{2}\right)\right],
$$

and the new metric function $F_{1}$ :

$$
\mathrm{e}^{F_{1}}=\mathrm{e}^{f_{1}}\left(1+\frac{r_{0}^{2}}{r^{2}}\right)^{-2} .
$$

Thus, the throat is located at $\eta=0$, and the solution for $\eta \leq 0$ is obtained from the solution for $\eta \geq 0$ by imposing the conditions $f_{0}(-\eta)=f_{0}(\eta), F_{1}(-\eta)=F_{1}(\eta)$ and $\phi(-\eta)=\phi(\eta)$. Since these conditions generically introduce jumps in the derivatives of the functions $f_{0}$ and $\phi$ at the throat, we impose the presence of a thin shell of matter at the throat.

The proper procedure here is the make use of an appropriate set of junction conditions [10,25], namely, we evaluate the jumps in the Einstein and scalar field equations that arise for $\eta \rightarrow-\eta$ :

$$
\left\langle G^{\mu}{ }_{\nu}-T^{\mu}{ }_{\nu}\right\rangle=s^{\mu}{ }_{\nu}, \quad\left\langle\nabla^{2} \phi+\dot{F} \mathcal{I}\right\rangle=s_{\text {scal }} .
$$

Here, the stress energy tensor of the matter at the throat has been denoted by $s^{\mu}{ }_{\nu}$, while the source term for the scalar field has been named $s_{\text {scal }}$, and $d F(\phi) / d \phi=\dot{F}$ denotes the derivative of the coupling function. Since the thin shells should be composed of ordinary (non-exotic) matter, we assume a perfect fluid at the throat with pressure $p$ and energy density $\epsilon_{c}$. Moreover, we assume a scalar charge density $\rho_{\text {scal }}$ together with a gravitational source:

$$
S_{\Sigma}=\int\left[\lambda_{1}+2 \lambda_{0} F(\phi) \bar{R}\right] \sqrt{-\bar{h}} \mathrm{~d}^{3} x
$$

employed before for EsGB wormholes without NUT charge [2,27,28]. Here $\lambda_{1}$ and $\lambda_{0}$ are constants, $\bar{h}_{a b}$ is the induced metric at the throat, and $\bar{R}$ the corresponding Ricci scalar. The junction conditions are obtained by substituting the metric into the set of equations (22).

Now, we evaluate the junction conditions for both invariants successively. Note that all functions and derivatives are evaluated at the throat. For the Gauss-Bonnet invariant, we obtain

$$
\begin{aligned}
\frac{4}{\eta_{0}^{2}} \dot{F} \phi^{\prime}\left(\eta_{0}^{2} \mathrm{e}^{-\frac{3}{2} F_{1}}+3 N^{2} \mathrm{e}^{f_{0}-\frac{5}{2} F_{1}}\right) & =\lambda_{1} \eta_{0}^{2}+4 \lambda_{0} F \frac{\eta_{0}^{2} \mathrm{e}^{-F_{1}}+3 N^{2} \mathrm{e}^{f_{0}-2 F_{1}}}{\eta_{0}^{2}}-\epsilon_{c} \eta_{0}^{2}, \\
N \cos \theta\left[\eta_{0}^{2} f_{0}^{\prime} \mathrm{e}^{-\frac{F_{1}}{2}}-8 \dot{F} \phi^{\prime}\left(\mathrm{e}^{-\frac{3}{2} F_{1}}+\frac{4 N^{2}}{\eta_{0}^{2}} \mathrm{e}^{f_{0}-\frac{5}{2} F_{1}}\right)\right] & =2 N \cos \theta\left[\left(\epsilon_{c}+p\right) \eta_{0}^{2}-4 \lambda_{0} F \frac{\eta_{0}^{2} \mathrm{e}^{-F_{1}}+4 N^{2} \mathrm{e}^{f_{0}-2 F_{1}}}{\eta_{0}^{2}}\right], \\
\frac{\eta_{0}^{2} f_{0}^{\prime}}{2} \mathrm{e}^{-\frac{F_{1}}{2}}-\frac{4 N^{2}}{\eta_{0}^{2}} \dot{F} \phi^{\prime} \mathrm{e}^{f_{0}-\frac{5}{2} F_{1}} & =p \eta_{0}^{2}+\lambda_{1} \eta_{0}^{2}-4 \lambda_{0} N^{2} F \frac{\mathrm{e}^{f_{0}-2 F_{1}}}{\eta_{0}^{2}}, \\
\mathrm{e}^{-F_{1}} \phi^{\prime}-4 \frac{\dot{F}}{\eta_{0}^{4}} f_{0}^{\prime}\left(\eta_{0}^{2} \mathrm{e}^{-2 F_{1}}+3 N^{2} \mathrm{e}^{f_{0}-3 F_{1}}\right) & =-4 \lambda_{0} \frac{\dot{F}}{\eta_{0}^{4}}\left(\eta_{0}^{2} \mathrm{e}^{-F_{1}}+N^{2} \mathrm{e}^{f_{0}-2 F_{1}}\right)+\frac{\rho_{\text {scal }}}{2},
\end{aligned}
$$

where the prime denotes the derivative with respect to $\eta$. These conditions follow from the $\left({ }_{t}^{t}\right),\left({ }^{t} \varphi\right)$, and $\left({ }^{\varphi} \varphi\right)$ components of the Einstein equations and from the scalar field equation, respectively. The $\left({ }_{\theta}{ }_{\theta}\right)$ equation is equivalent to the $\left({ }^{\varphi} \varphi_{\varphi}\right)$ equation, all other equations are satisfied trivially. The $\theta$ dependence in the $\left({ }^{t}{ }_{\varphi}\right)$ equation factorizes, in fact, this equation is satisfied once the $\left({ }_{t}^{t}\right)$ and $\left({ }^{\varphi}{ }_{\varphi}\right)$ equations are solved.

A simple example would be pressureless matter, $p=0$. With

$$
\lambda_{0}=\frac{\dot{F}}{F} \mathrm{e}^{-\frac{F_{1}}{2}} \phi^{\prime}, \quad \lambda_{1}=\frac{f_{0}^{\prime}}{2} \mathrm{e}^{-\frac{F_{1}}{2}},
$$


we obtain

$$
\epsilon_{c}=\frac{f_{0}^{\prime}}{2} \mathrm{e}^{-\frac{F_{1}}{2}} .
$$

For all of our solutions $f_{0}^{\prime}>0$, therefore, the energy density $\epsilon_{c}$ is always positive for this choice of the constants $\lambda_{0}$ and $\lambda_{1}$.

For the Chern-Simons invariant, we obtain

$$
\begin{aligned}
8 N \dot{F} \phi^{\prime} f_{0}^{\prime} \mathrm{e}^{\frac{f_{0}}{2}} \mathrm{e}^{-2 F_{1}} & =\lambda_{1} \eta_{0}^{2}+4 \lambda_{0} F \frac{\eta_{0}^{2} \mathrm{e}^{-F_{1}}+3 N^{2} \mathrm{e}^{f_{0}-2 F_{1}}}{\eta_{0}^{2}}-\epsilon_{c} \eta_{0}^{2} \\
N \cos \theta f_{0}^{\prime}\left(\eta_{0}^{2} \mathrm{e}^{-\frac{F_{1}}{2}}-24 N \dot{F} \phi^{\prime} \mathrm{e}^{\frac{f_{0}}{2}-2 F_{1}}\right) & =2 N \cos \theta\left(\epsilon_{c}+p\right) \eta_{0}^{2}-8 N \cos \theta \lambda_{0} F \frac{\eta_{0}^{2} \mathrm{e}^{-F_{1}}+4 N^{2} \mathrm{e}^{f_{0}-2 F_{1}}}{\eta_{0}^{2}} \\
\frac{f_{0}^{\prime}}{2}\left(\eta_{0}^{2} \mathrm{e}^{-\frac{F_{1}}{2}}-8 N \dot{F} \phi^{\prime} \mathrm{e}^{\frac{f_{0}}{2}-2 F_{1}}\right) & =p \eta_{0}^{2}+\lambda_{1} \eta_{0}^{2}-4 \lambda_{0} N^{2} F \frac{\mathrm{e}^{f_{0}-2 F_{1}}}{\eta_{0}^{2}} \\
\mathrm{e}^{-F_{1}} \phi^{\prime}-4 N \frac{\dot{F}}{\eta_{0}^{2}}\left(f_{0}^{\prime}\right)^{2} \mathrm{e}^{\frac{f_{0}-5 F_{1}}{2}} & =-4 \lambda_{0} \frac{\dot{F}}{\eta_{0}^{4}}\left(\eta_{0}^{2} \mathrm{e}^{-F_{1}}+N^{2} \mathrm{e}^{f_{0}-2 F_{1}}\right)+\frac{\rho_{\mathrm{scal}}}{2}
\end{aligned}
$$

again from the $\left({ }_{t}^{t}\right),\left({ }^{t}{ }_{\varphi}\right),\left({ }^{\varphi}{ }_{\varphi}\right)$ components of the Einstein equations and the scalar field equation, respectively.

Considering again pressureless matter, $p=0$, with

$$
\lambda_{0}=\frac{\eta_{0}^{2} \dot{F}}{N F} \mathrm{e}^{-\frac{f_{0}}{2}}, \quad \lambda_{1}=-\frac{f_{0}^{\prime}}{2} \mathrm{e}^{-\frac{F_{1}}{2}},
$$

we now obtain

$$
\epsilon_{c}=\frac{f_{0}^{\prime}}{2 N}\left(N \mathrm{e}^{-\frac{F_{1}}{2}}+8 \dot{F} \phi^{\prime} \mathrm{e}^{-\frac{f_{0}}{2}-F_{1}}\right)+\frac{4 N}{\eta_{0}^{2}} \dot{F} \phi^{\prime} f_{0}^{\prime} \mathrm{e}^{\frac{f_{0}}{2}-2 F_{1}}
$$

\section{Energy conditions}

To obtain wormhole solutions, the null energy condition (NEC):

$$
T_{\mu \nu} n^{\mu} n^{\nu} \geq 0
$$

must be violated. Here $n^{\mu}$ may be any null vector $\left(n^{\mu} n_{\mu}=0\right)$. Consequently, all one has to show is that null vectors exist, such that $T_{\mu \nu} n^{\mu} n^{\nu}<0$ somewhere in the spacetime. Choosing the null vector $n^{\mu}$

$$
n^{\mu}=\left(1, \sqrt{-g_{t t} / g_{\eta \eta}}, 0,0\right)
$$

and thus $n_{\mu}=\left(g_{t t}, \sqrt{-g_{t t} g_{\eta \eta}}, 0,0\right)$, the NEC leads to

$$
T_{\mu \nu} n^{\mu} n^{\nu}=T_{t}^{t} n^{t} n_{t}+T_{\eta}^{\eta} n^{\eta} n_{\eta}=-g_{t t}\left(-T_{t}^{t}+T_{\eta}^{\eta}\right) .
$$

This shows that the NEC is violated when

$$
-T_{t}^{t}+T_{\eta}^{\eta}<0
$$

Alternatively, choosing the null vector

$$
n^{\mu}=\left(1,0, \sqrt{-g_{t t} / g_{\theta \theta}}, 0\right)
$$

leads to NEC violation when

$$
-T_{t}^{t}+T_{\theta}^{\theta}<0 .
$$

These conditions have been investigated before for various wormhole solutions (see, e.g., [2,27, 28,34]). 


\section{Results}

We solve the coupled Einstein and scalar field equations numerically. To this end we introduce the inverse radial coordinate $x=1 / r$. Consequently, the asymptotic region $r \rightarrow \infty$ corresponds to the region $x \rightarrow 0$. Here the expansion of the metric functions and the scalar field then reads (Eqs. (17)-(19)):

$$
f_{0}=-2 M x+\mathcal{O}\left(x^{3}\right), \quad f_{1}=2 M x+\mathcal{O}\left(x^{2}\right), \quad \phi=\phi_{\infty}-D x+\mathcal{O}\left(x^{3}\right) .
$$

To solve the system of ODEs, we treat it as an initial value problem, and employ the fourth order Runge Kutta method. The above expansion then yields the initial values:

$$
f_{0, \text { ini }}=0, \quad f_{0, \text { ini }}^{\prime}=-2 M, \quad f_{1, \text { ini }}=0, \quad f_{1, \text { ini }}^{\prime}=2 M, \quad \phi_{\text {ini }}=0, \quad \phi_{\text {ini }}^{\prime}=-D,
$$

where the prime denotes the derivative with respect to $x$. We start the computation at spatial infinity, $x=0$, and end at the throat at some finite value $x=x_{0}$, where we reach the condition (14).

By following the outlined numerical procedure, we have constructed numerous sets of wormhole solutions with NUT charge for both invariants $\mathcal{I}(g)$. In the following, we demonstrate our results for both invariants successively.

\subsection{Case (i): EsGB wormholes}

We begin by showing a typical solution for EsGB wormholes with NUT charge, where we have chosen the scaled coupling constant $\alpha / M^{2}=2.5$, the scaled scalar charge $D / M=2$ and the scaled NUT charge $n=N / M=3$. The metric functions $\mathrm{e}^{f_{0}}$ and $\mathrm{e}^{F_{1}}$, and the scalar field function $\phi$ are shown in Fig. 1a vs the scaled radial coordinate $\eta / M$. Also shown is the scaled circumferential radius $R_{c} / \eta_{0}$ vs $\eta / M$, where $\eta_{0} / M=0.517$. The minimum of $R_{c}$ at the coordinate origin $\eta=0$ corresponds to the location of the throat.

As required the wormhole solutions violate the energy conditions. We demonstrate the violation of the NEC conditions $-T_{t}^{t}+T_{\eta}^{\eta} \geq 0$ and $-T_{t}^{t}+T_{\theta}^{\theta} \geq 0$ for the same solution in Fig. 1b. Clearly, both expressions are negative in a region close to the throat. Note that we have omitted in the notation of the energy conditions that we refer to the effective stress energy tensor. We emphasize again that the violation is caused by the gravitational part of the effective stress energy tensor.

We next turn to the domain of existence of these EsGB wormholes with NUT charge. The domain of existence is shown in terms of the scaled coupling constant $8 \alpha / M^{2}$ and the scaled scalar charge $D / M$ in Fig. 1c for several values of the scaled NUT charge, $n=N / M=1,2$, and 3, and compared with the case of vanishing NUT charge, $n=0$ [2]. Note that we only show the right hand side $D \geq 0$ of the domain, since the domain is symmetric with respect to $D \rightarrow-D$, which is a consequence of the quadratic coupling function and the chosen boundary condition $\phi_{\infty}=0$.

For any given value of the NUT charge, the domain of existence is delimited by three sets of solutions. The first set corresponds to the scalarized black holes with NUT charge of Brihaye et at. [5], which we fully reproduce. These black holes are shown by a solid green curve. The second set is shown by a dotted red curve and corresponds to a set of singular solutions. Here a cusp singularity is encountered at some value $\eta_{\star}$ of the radial coordinate. Cusp singularities are a recurring feature of scalarized wormholes (see $[2,23,29,30])$. They form, when the determinant of the coefficients of the second order terms of the ODEs vanishes.

The third set of boundary solutions is shown by a dashed blue curve and referred to as degenerate set of solutions. To clarify the physical meaning of this set, we recall that we identified the throat by looking for a minimum of the circumferential radius. However, it may happen that this extremum becomes a degenerate extremum. Here, in principle, a new set of wormholes emerges, which possess an equator and a double throat, i.e., the degenerate extremum will split into a maximum (equator) and two minima (throats). We remark, that for $D=0$ the scalar field vanishes, thus vacuum solutions of General Relativity are recovered. Overall we note that with increasing NUT charge the domain of existence of wormhole solutions increases rapidly.

We now turn to the causal structure of the EsGB wormhole spacetimes. The relevant quantity to consider here is the polar angle, where the $g_{\varphi \varphi}$ component changes sign, and thus closed time-like curves will be allowed. In particular, we are interested in the critical polar angle $\theta_{c}$, where this change of sign occurs at the wormhole throat. We show the boundary of the domain with changed causal structure at the throat in Fig. 1d, where we exhibit the critical polar angle $\theta_{c}$ vs the scaled coupling constant $8 \alpha / M^{2}$ for several values of the scaled NUT charge, $n=N / M=1,2$, and 3. For a fixed value of the NUT charge the dotted blue curve shows the singular 


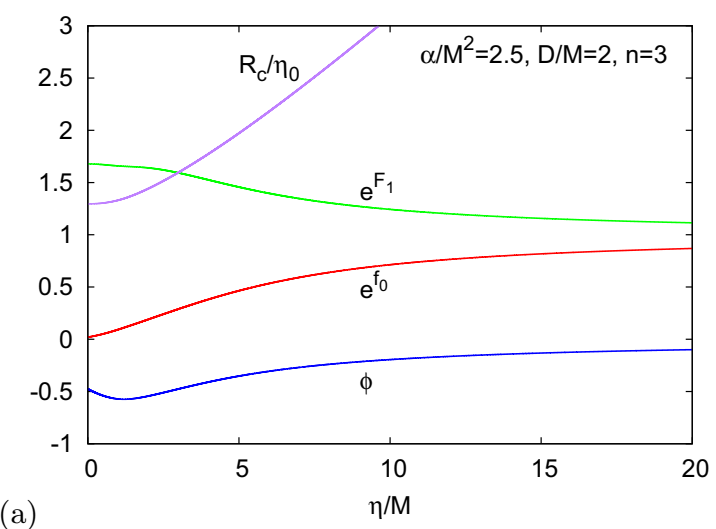

(a)

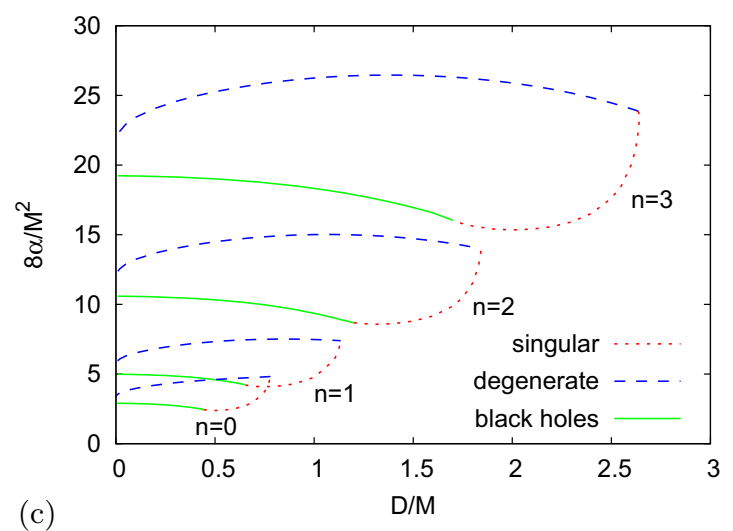

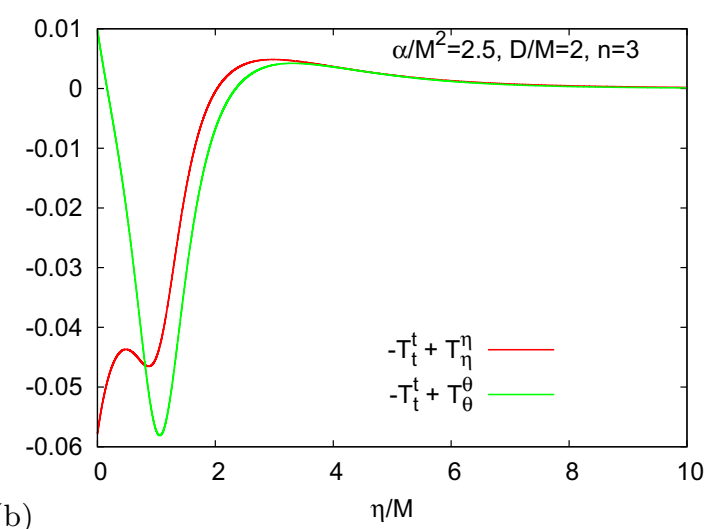

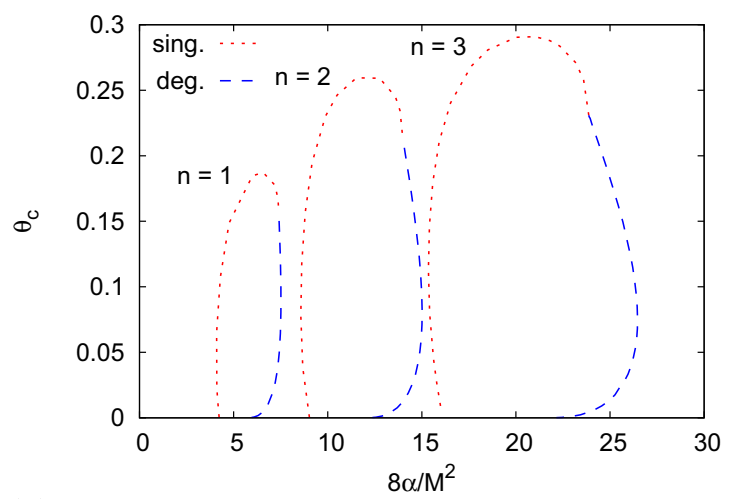

(d)

Fig. 1 EsGB wormhole solutions with NUT charge: a metric profile functions $\mathrm{e}^{f_{0}}, \mathrm{e}^{F_{1}}$, scalar field function $\phi$, and scaled circumferential radius $R_{c} / \eta_{0}$ vs radial coordinate (parameters $\alpha / M^{2}=2.5, D / M=2$ and $n=N / M=3$ ); $\mathbf{b}$ NEC conditions $-T_{t}^{t}+T_{\eta}^{\eta} \geq 0$ and $-T_{t}^{t}+T_{\theta}^{\theta} \geq 0$ vs radial coordinate $\eta$ (parameters $\alpha / M^{2}=2.5, D / M=2$ and $n=N / M=3$ ); c domain of existence for several values of the scaled NUT charge $n=N / M$ : scaled coupling constant $8 \alpha / M^{2}$ vs scaled scalar charge $D / M$ (solid green: black hole limit, dashed blue: degenerate wormhole limit, dotted red: singular limit); d critical angle $\theta_{c}$ vs scaled coupling constant $8 \alpha / M^{2}$ (dashed blue: degenerate wormhole limit, dotted red: singular limit)

boundary set, while the dashed green curve shows the degenerate boundary set. Only for the limiting case of black holes the critical polar angle $\theta_{c}$ goes to zero. Thus all the wormholes with NUT charge possess a region with closed time-like curves that extends to their throat.

\subsection{Case (ii): EsCS wormholes}

We now discuss the properties of EsCS wormholes with NUT charge. We start again with a typical wormhole solution, that has been obtained for the parameter choice $\alpha / M^{2}=4, D / M=1$ and $n=N / M=3$. We show its metric functions $\mathrm{e}^{f_{0}}$ and $\mathrm{e}^{F_{1}}$, and its scalar field function $\phi$ vs the scaled radial coordinate $\eta / M$ in Fig. 2a, along with its scaled circumferential radius $R_{c} / \eta_{0}$, where $\eta_{0} / M=0.495$. We note that the functions are rather similar to those of the EsGB wormholes shown in Fig. 1a.

We demonstrate the violation of the NEC conditions $-T_{t}^{t}+T_{\eta}^{\eta} \geq 0$ and $-T_{t}^{t}+T_{\theta}^{\theta} \geq 0$ in Fig. $2 \mathrm{~b}$ for this solution. Again we find a region close to the throat, where both conditions are violated.

The domain of existence of the EsCS wormholes is shown in terms of the scaled coupling constant $8 \alpha / M^{2}$ and the scaled scalar charge $D / M$ in Fig. 2c for several values of the scaled NUT charge, $n=N / M=0.5$, 1,2 , and 3 . The boundaries of the domain of existence are completely analogous to the previously discussed case of EsGB wormholes with NUT charge.

The first set of boundary solutions corresponds to the scalarized black holes with NUT charge of Brihaye et al. [5] and is shown by a solid green curve. The second set of boundary solutions corresponds to the set of solutions with a cusp singularity and is shown by a dotted red curve. Finally the third set of boundary solutions 

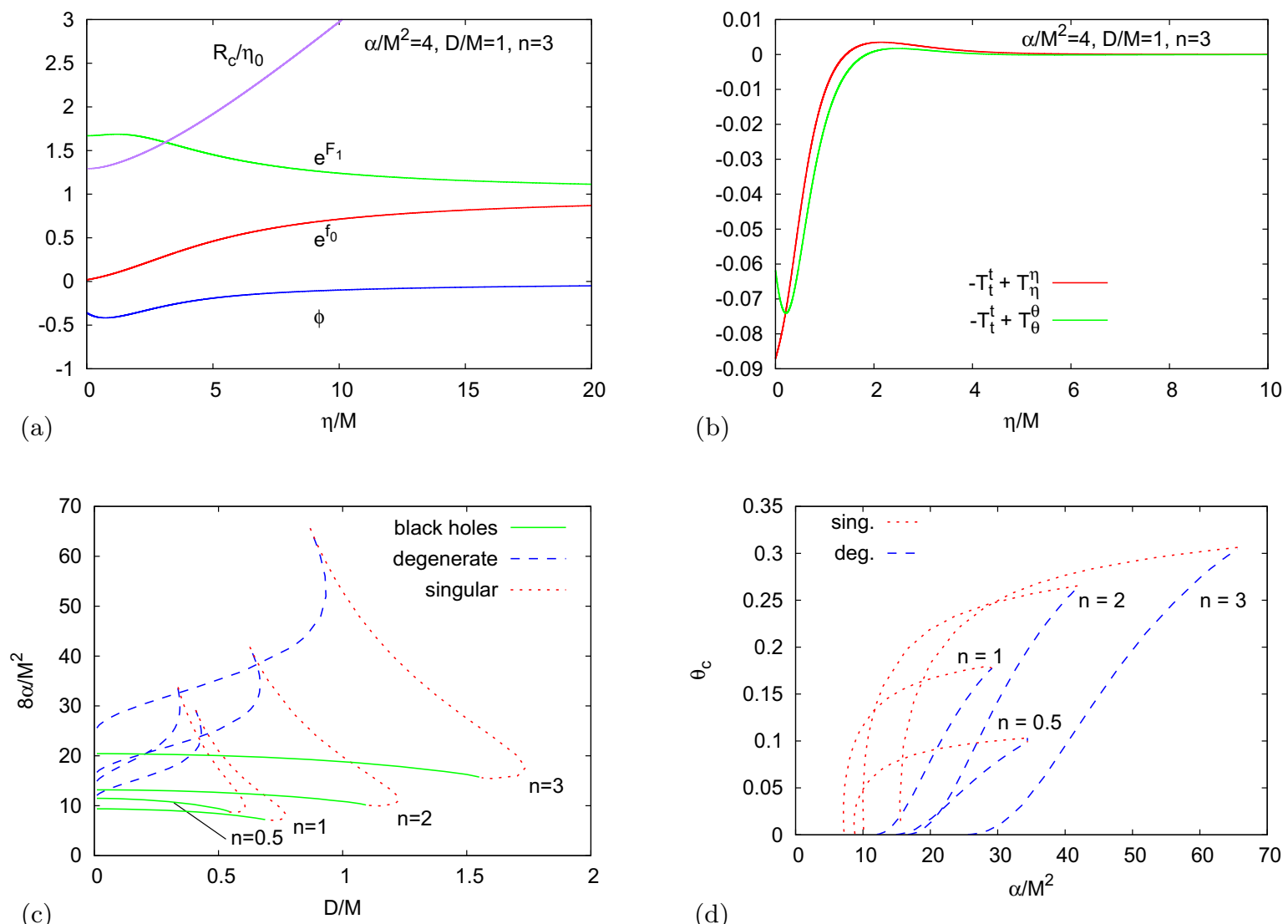

(d)

Fig. 2 EsCS wormhole solutions with NUT charge: a metric profile functions $\mathrm{e}^{f_{0}}, \mathrm{e}^{F_{1}}$, scalar field function $\phi$, and scaled circumferential radius $R_{c} / \eta_{0}$ vs radial coordinate (parameters $\alpha / M^{2}=4, D / M=1$ and $n=N / M=3$ ); b NEC conditions $-T_{t}^{t}+T_{\eta}^{\eta} \geq 0$ and $-T_{t}^{t}+T_{\theta}^{\theta} \geq 0$ vs radial coordinate $\eta$ (parameters $\alpha / M^{2}=4, D / M=1$ and $n=N / M=3$ ); c domain of existence for several values of the scaled NUT charge $n=N / M$ : scaled coupling constant $8 \alpha / M^{2}$ vs scaled scalar charge $D / M$ (solid green: black hole limit, dashed blue: degenerate wormhole limit, dotted red: singular limit); d critical angle $\theta_{c}$ vs scaled coupling constant $8 \alpha / M^{2}$ (dashed blue: degenerate wormhole limit, dotted red: singular limit)

corresponds to wormhole solutions with a degenerate throat, beyond which wormholes with an equator and a double throat arise. They are shown by a dashed blue curve. The domain of existence is again symmetric with respect to $D \rightarrow-D$, and for $D=0$ solutions of General Relativity are recovered.

However, we also notice a new feature of the EsCS wormholes as compared to the EsGB wormholes. Whereas for EsGB wormholes the domain of existence moves continuously to smaller values of the coupling constant $\alpha$, as the NUT charge is decreased towards zero, this is not the case for the EsCS wormholes. Here the domain of existence exhibits the same dependence as the scalarized EsCS black holes, namely, after reaching a minimum of $\alpha$ with decreasing NUT charge, a further decease of the NUT charge leads to increasing values of $\alpha$. This is seen in Fig. 2c, where the domain of existence for $n=0.5$ has moved to larger values of $\alpha$ as compared to the domain for $n=1$. As the NUT charge tends towards zero, the coupling constant $\alpha$ tends towards infinity. However, in the limit a finite source term for the scalar field results, since the NUT charge and the coupling constant conspire appropriately.

We finally turn to the causal structure of the EsCS wormhole spacetimes. We show the critical polar angle $\theta_{c}$, where the metric component $g_{\varphi \varphi}$ changes sign at the wormhole throat, vs the scaled coupling constant $8 \alpha / M^{2}$ in Fig. $2 \mathrm{~d}$ for the same set of values of the scaled NUT charge, $n=N / M=0.5,1,2$, and 3. Again, for a fixed NUT charge, the boundaries are given by the dotted blue curve showing the singular boundary set, and the dashed green curve showing the degenerate boundary set. Only for the limiting case of black holes the critical polar angle $\theta_{c}$ goes to zero. 


\section{Conclusions}

We have considered scalarized wormholes with NUT charge in two alternative gravity theories, EsGB theory and EsCS theory. In both cases a scalar field has been coupled with a quadratic coupling function to the respective invariant, since this choice of coupling function allows for spontaneously scalarized black holes with NUT charge, i.e., solutions obtained before by Brihaye et al. [5].

In these alternative gravity theories, wormholes arise without the need of introducing exotic matter. The reason is the presence of gravitational terms in the stress energy tensor that result, respectively, from the higher curvature GB term or CS term. These terms allow for violations of the energy conditions and, therefore, the presence of wormholes.

We have mapped out the domain of existence of these wormholes for various values of the NUT charge. In all cases, for a given NUT charge, the domain has one boundary consisting of scalarized black holes with NUT charge, one boundary formed by solutions with a cusp singularity, and one boundary composed of wormholes with a degenerate throat, where a new type of wormhole arises, that features an equator and a double throat, i.e., wormholes to be addressed in the future.

To have symmetric wormholes without singularities (apart from the Misner string caused by the NUT charge), we have cut the wormhole spacetimes at the throat, retaining only the part extending to infinity, and pasted the symmetrically reflected copy at the throat. We have shown that the resulting junction conditions at the throat can be fulfilled by a thin shell of ordinary matter, such as dust, for example.

Since these wormholes carry NUT charge, they possess closed time-like curves. Therefore, we have investigated, in particular, the causal structure at the throat. Interestingly, all these wormholes with NUT charge possess closed time-like curves that extend all the way to the throat. However, for the limiting set of scalarized black holes with NUT charge the closed time-like curves do not extend to the horizon, but can get arbitrarily close to it.

We have considered wormholes with a NUT charge as a toy model for rotating wormholes in EsGB theory and EsCS theory. In this toy model the angular dependence factorizes, leaving a set of nonlinear coupled ODEs to be solved numerically. In the case of rotating wormholes, we will have to solve sets of nonlinear coupled partial differential equations, which will be rather challenging for these alternative gravity theories. Moreover, the proper conditions for the throat and the proper set of junction conditions will have to be formulated and solved in this case, as well.

Acknowledgements BK and JK gratefully acknowledge support by the DFG Research Training Group 1620 Models of Gravity and the COST Actions CA15117 and CA16104.

Open Access This article is licensed under a Creative Commons Attribution 4.0 International License, which permits use, sharing, adaptation, distribution and reproduction in any medium or format, as long as you give appropriate credit to the original author(s) and the source, provide a link to the Creative Commons licence, and indicate if changes were made. The images or other third party material in this article are included in the article's Creative Commons licence, unless indicated otherwise in a credit line to the material. If material is not included in the article's Creative Commons licence and your intended use is not permitted by statutory regulation or exceeds the permitted use, you will need to obtain permission directly from the copyright holder. To view a copy of this licence, visit http://creativecommons.org/licenses/by/4.0/.

\section{References}

1. Antoniou, G.; Bakopoulos, A.; Kanti, P.: Evasion of no-hair theorems and novel black-hole solutions in Gauss-Bonnet theories. Phys. Rev. Lett. 120, 131102 (2018)

2. Antoniou, G.; Bakopoulos, A.; Kanti, P.; Kleihaus, B.; Kunz, J.: Novel Einstein-scalar-Gauss-Bonnet wormholes without exotic matter. Phys. Rev. D 101(2), 024033 (2020)

3. Berti, E.; Barausse, E.; Cardoso, V.; Gualtieri, L.; Pani, P.; Sperhake, U.; Stein, L.C.; Wex, N.; Yagi, K.; Baker, T.; et al.: Testing general relativity with present and future astrophysical observations. Class. Quantum Gravity 32, 243001 (2015)

4. Berti, E.; Collodel, L.G.; Kleihaus, B.; Kunz, J.: Spin-induced black-hole scalarization in Einstein-scalar-Gauss-Bonnet theory. Phys. Rev. Lett. 126(1), 011104 (2021)

5. Brihaye, Y.; Herdeiro, C.; Radu, E.: The scalarised Schwarzschild-NUT spacetime. Phys. Lett. B 788, 295 (2019)

6. Bronnikov, K.A.: Scalar-tensor theory and scalar charge. Acta Phys. Pol. B 4, 251 (1973)

7. Bronnikov, K.A.; Elizalde, E.: Wormhole geometries in $\mathrm{f}(\mathrm{R})$ modified theories of gravity. Phys. Rev. D 81, 044032 (2010)

8. Collodel, L.G.; Kleihaus, B.; Kunz, J.; Berti, E.: Spinning and excited black holes in Einstein-scalar-Gauss-Bonnet theory. Class. Quantum Gravity 37, 075018 (2020)

9. Cunha, P.V.P.; Herdeiro, C.A.R.; Radu, E.: Spontaneously scalarized Kerr black holes in extended scalar-tensor-GaussBonnet Gravity. Phys. Rev. Lett. 123, 011101 (2019)

10. Davis, S.C.: Generalized Israel junction conditions for a Gauss-Bonnet brane world. Phys. Rev. D 67, 024030 (2003) 
11. Delsate, T.; Herdeiro, C.; Radu, E.: Non-perturbative spinning black holes in dynamical Chern-Simons gravity. Phys. Lett. B 787, 8-15 (2018)

12. Dima, A.; Barausse, E.; Franchini, N.; Sotiriou, T.P.: Spin-induced black hole spontaneous scalarization. Phys. Rev. Lett. 125, 231101 (2020)

13. Doneva, D.D.; Yazadjiev, S.S.: New Gauss-Bonnet black holes with curvature induced scalarization in the extended scalartensor theories. Phys. Rev. Lett. 120, 131103 (2018)

14. Ellis, H.G.: Ether flow through a drainhole—a particle model in general relativity. J. Math. Phys. 14, 104 (1973)

15. Ellis, H.G.: The evolving, flowless drain hole: a nongravitating particle model in general relativity theory. Gen. Relativ. Gravit. 10, 105 (1979)

16. Faraoni, V.; Capozziello, S.: Beyond Einstein Gravity: A Survey of Gravitational Theories for Cosmology and Astrophysics. Springer, Dordrecht (2011)

17. Fukutaka, H.; Tanaka, K.; Ghoroku, K.: Wormhole solutions in higher derivative gravity. Phys. Lett. B 222, 191 (1989)

18. Furey, N.; DeBenedictis, A.: Wormhole throats in Rm gravity. Class. Quantum Gravity 22, 313 (2005)

19. Ghoroku, K.; Soma, T.: Lorentzian wormholes in higher derivative gravity and the weak energy condition. Phys. Rev. D 46, 1507 (1992)

20. Harko, T.; Lobo, F.S.N.; Mak, M.K.; Sushkov, S.V.: Modified-gravity wormholes without exotic matter. Phys. Rev. D 87, 067504 (2013)

21. Herdeiro, C.A.R.; Radu, E.; Silva, H.O.; Sotiriou, T.P.; Yunes, N.: Spin-induced scalarized black holes. Phys. Rev. Lett. 126(1), $011103(2021)$

22. Hochberg, D.: Lorentzian wormholes in higher order gravity theories. Phys. Lett. B 251, 349 (1990)

23. Ibadov, R.; Kleihaus, B.; Kunz, J.; Murodov, S.: Wormholes in Einstein-scalar-Gauss-Bonnet theories with a scalar selfinteraction potential. Phys. Rev. D 102, 064010 (2020)

24. Ibadov, R.; Kleihaus, B.; Kunz, J.; Murodov, S.: Scalarized nutty wormholes. Symmetry 13(1), 89 (2021)

25. Israel, W.: Singular hypersurfaces and thin shells in general relativity. Nuovo Cim. B 44S10, 1 (1966) [Erratum: Nuovo Cim. B 48, 463 (1967)]

26. Kagramanova, V.; Kunz, J.; Hackmann, E.; Lammerzahl, C.: Analytic treatment of complete and incomplete geodesics in Taub-NUT space-times. Phys. Rev. D 81, 124044 (2010)

27. Kanti, P.; Kleihaus, B.; Kunz, J.: Wormholes in dilatonic Einstein-Gauss-Bonnet theory. Phys. Rev. Lett. 107, 271101 (2011)

28. Kanti, P.; Kleihaus, B.; Kunz, J.: Stable Lorentzian wormholes in dilatonic Einstein-Gauss-Bonnet theory. Phys. Rev. D 85, 044007 (2012)

29. Kleihaus, B.; Kunz, J.; Kanti, P.: Particle-like ultracompact objects in Einstein-scalar-Gauss-Bonnet theories. Phys. Lett. B 804, $135401(2020)$

30. Kleihaus, B.; Kunz, J.; Kanti, P.: Properties of ultracompact particlelike solutions in Einstein-scalar-Gauss-Bonnet theories. Phys. Rev. D 102(2), 024070 (2020)

31. Kodama, T.: General relativistic nonlinear field: a kink solution in a generalized geometry. Phys. Rev. D 18, 3529 (1978)

32. Lobo, F.S.: Introduction. Fundam. Theor. Phys. 189, 1 (2017)

33. Lobo, F.S.N.; Oliveira, M.A.: Wormhole geometries in $\mathrm{f}(\mathrm{R})$ modified theories of gravity. Phys. Rev. D 80, 104012 (2009)

34. Morris, M.S.; Thorne, K.S.: Wormholes in space-time and their use for interstellar travel: a tool for teaching general relativity. Am. J. Phys. 56, 395 (1988)

35. Saridakis, E.N.; et al. [CANTATA]: Modified Gravity and Cosmology: An Update by the CANTATA Network. arXiv:2105.12582 [gr-qc]

36. Silva, H.O.; Sakstein, J.; Gualtieri, L.; Sotiriou, T.P.; Berti, E.: Spontaneous scalarization of black holes and compact stars from a Gauss-Bonnet coupling. Phys. Rev. Lett. 120, 131104 (2018)

37. Visser, M.: Lorentzian Wormholes: From Einstein to Hawking. AIP, Woodbury (1995)

38. Will, C.M.: The confrontation between general relativity and experiment. Living Rev. Relativ. 9,3 (2006)

39. Yunes, N.; Pretorius, F.: Dynamical Chern-Simons modified gravity. I. Spinning black holes in the slow-rotation approximation. Phys. Rev. D 79, 084043 (2009)

Publisher's Note Springer Nature remains neutral with regard to jurisdictional claims in published maps and institutional affiliations. 